\title{
Genomic analyses provide insights into breed-of-origin effects from purebreds on three-way crossbred pigs
}

\author{
Yu Lin Equal first author, ${ }^{1}$, Qianzi Tang Equal first author, 1 , Yan $\mathrm{Li}^{1}{ }^{1}$, Mengnan He $^{1}$, Long Jin ${ }^{1}$, Jideng Ma ${ }^{1}$, Xun Wang ${ }^{1}$, Keren Long \\ ${ }^{1}$, Zhiqing Huang ${ }^{2}$, Xuewei $\mathbf{L i}^{1}$, Yiren $\mathbf{G u}^{3}$, Mingzhou Li ${ }^{\text {Corresp. } 1}$ \\ 1 Institute of Animal Genetics and Breeding, College of Animal Science and Technology, Sichuan Agricultural University, Chengdu, Sichuan, China \\ 2 Institute of Animal Nutrition, Sichuan Agricultural University, Chengdu, Sichuan, China \\ 3 Sichuan Animal Science Academy, Chengdu, Sichuan, China \\ Corresponding Author: Mingzhou Li \\ Email address: mingzhou.li@sicau.edu.cn
}

Crossbreeding is widely used aimed at improving crossbred performance for poultry and livestock. Alleles that are specific to different purebreds will yield a large number of heterozygous single-nucleotide polymorphisms (SNPs) in crossbred individuals, which are supposed to have the power to alter gene function or regulate gene expression. For pork production, a classic three-way crossbreeding system of Duroc $\times$ (Landrace $\times$ Yorkshire) (DLY) is generally used to produce terminal crossbred pigs with stable and prominent performance. Nonetheless, little is known about the breed-of-origin effects from purebreds on DLY pigs. In this study, we first estimated the distribution of heterozygous SNPs in three kinds of three-way crossbred pigs via whole genome sequencing data originated from three purebreds. The result suggested that DLY is a more effective strategy for three-way crossbreeding as it could yield more stably inherited heterozygous SNPs. We then sequenced a DLY pig family and identified 95, 79, 132 and 42 allele-specific expression (ASE) genes in adipose, heart, liver and skeletal muscle, respectively. Principal component analysis (PCA) and unrestricted clustering analyses revealed the tissue-specific pattern of ASE genes, indicating the potential roles of ASE genes for development of DLY pigs. In summary, our findings provided a lot of candidate SNP markers and ASE genes for DLY three-way crossbreeding system, which may be valuable for pig breeding and production in the future. 
1 Genomic analyses provide insights into breed-of-origin effects from

2 purebreds on three-way crossbred pigs

3 Yu Lin ${ }^{1 \dagger}$, Qianzi Tang ${ }^{1 \dagger}$, Yan Li ${ }^{1}$, Mengnan He${ }^{1}$, Long Jin ${ }^{1}$, Jideng Ma ${ }^{1}$, Xun Wang ${ }^{1}$, Keren

4 Long ${ }^{1}$, Zhiqing Huang ${ }^{2}$, Xuewei Li ${ }^{1}$, Yiren Gu${ }^{3}$, Mingzhou Li1*

5 1. Institute of Animal Genetics and Breeding, College of Animal Science and Technology,

6 Sichuan Agricultural University, Chengdu 611130, China

7 2. Institute of Animal Nutrition, Sichuan Agricultural University, Chengdu 611130, China

8 3. Sichuan Animal Science Academy, Chengdu 610066, China

$9 \quad{ }^{*}$ Corresponding Author: mingzhou.li@sicau.edu.cn

10 These authors contributed equally to this work

11 Abstract

12 Crossbreeding is widely used aimed at improving crossbred performance for poultry and

13 livestock. Alleles that are specific to different purebreds will yield a large number of

14 heterozygous single-nucleotide polymorphisms (SNPs) in crossbred individuals, which are

15 supposed to have the power to alter gene function or regulate gene expression. For pork

16 production, a classic three-way crossbreeding system of Duroc $\times$ (Landrace $\times$ Yorkshire) $($ DLY) 
17 is generally used to produce terminal crossbred pigs with stable and prominent performance.

18 Nonetheless, little is known about the breed-of-origin effects from purebreds on DLY pigs. In

19 this study, we first estimated the distribution of heterozygous SNPs in three kinds of three-way

20 crossbred pigs via whole genome sequencing data originated from three purebreds. The result

21 suggested that DLY is a more effective strategy for three-way crossbreeding as it could yield

22 more stably inherited heterozygous SNPs. We then sequenced a DLY pig family and identified

$2395,79,132$ and 42 allele-specific expression (ASE) genes in adipose, heart, liver and skeletal

24 muscle, respectively. Principal component analysis (PCA) and unrestricted clustering analyses

25 revealed the tissue-specific pattern of ASE genes, indicating the potential roles of ASE genes for

26 development of DLY pigs. In summary, our findings provided a lot of candidate SNP markers

27 and ASE genes for DLY three-way crossbreeding system, which may be valuable for pig

28 breeding and production in the future.

\section{Keywords}

30 Crossbreeding, breed-of-origin, SNP, DLY, allele-specific expression

31 Introduction

32 Crossbreeding strategy for animals (especially agricultural poultry and livestock) is a classic and 
33 effective method aimed at improving crossbred performance (Sevillano et al., 2016; Sheng et al.,

34 2013; Zhang et al., 2018). Using crossbreeding strategy, alleles that are specific to different

35 purebreds will be inherited by offspring and thereby yield a large number of heterozygous SNPs

36 (Sevillano et al., 2016; Vandenplas et al., 2016). These heterozygous SNPs are supposed to have

37 a contribution on improving crossbred performance, especially in terms of growth rate,

38 reproductive performance, production performance (egg, meat and milk) and disease resistance

39 (Puppel et al., 2018; Ragab et al., 2016; Rinell \& Heringstad 2018).

Pigs (Sus scrofa) were domesticated at least $\sim 9,000$ years ago and have been used as a major

41 source of animal proteins in the human diet (Chen et al., 2007). There are over 730 distinct pig

42 breeds worldwide (Chen et al., 2007). The worldwide distribution of pigs is dominated by six international transboundary commercial pig breeds originating in Europe, namely, Berkshire,

44 Duroc, Hampshire, Landrace, Piétrain, and Yorkshire, of which Duroc and Hampshire pigs were

45 developed mainly in North America (Chen et al., 2007). After the long-term practice for the

46 presence of combinations of abilities, a terminal crossbreeding system with three pig breeds,

47 namely, Duroc $\times($ Landrace $\times$ Yorkshire $)($ DLY $)$, is generally used for commercial pork

48 production (Hua et al., 2014; Liu et al., 2015). Landrace and Yorkshire pigs share prominent 
49 traits for pork production, typically, long carcass length, thin subcutaneous fat layer, large hams,

50 good mothering ability, and high muscularity in the carcass (Choi et al., 2016; Ruusunen et al.,

51 2012). Duroc pigs are mainly used to enhance the growth rate and intramuscular fat in this three-

52 way crossbreeding system (Choi et al., 2016). The pigs generated by this system exhibit a

53 collection of excellent traits, such as high productivity, rapid growth, desirable pork quality and

54 pork production (Choi et al., 2016).

55 Though previous studies have focused on ASE genes to investigate the breed-of-origin

56 effects from purebreds on crossbred individuals for bacterial resistance (Wu et al., 2015),

57 peripheral blood (Maroilley et al., 2017), brain development (Oczkowicz et al., 2018), prenatal

58 skeletal muscle development (Yang et al., 2018), adipogenesis and lipid metabolism (Stachowiak

59 et al., 2018), such studies are absent for DLY three-way crossbred pigs. Here, we first estimated

60 the distribution of heterozygous SNPs in three kinds of three-way crossbred pigs via whole

61 genome sequencing data originated from three purebreds (including 11 Duroc, 9 Landrace and

6210 Yorkshire pigs). Based on this estimation, we suggested that DLY is a more effective strategy

63 for three-way crossbreeding system among Duroc, Landrace, and Yorkshire breeds as it could

64 generate more stably inherited heterozygous SNPs. We then sequenced a DLY pig family and 
65 identified 95, 79, 132 and 42 ASE genes in adipose, heart, liver and skeletal muscle, respectively.

66 PCA and unrestricted clustering analysis revealed that these ASE genes were mainly tissue-

67 specific, suggesting the potential roles of ASE genes during the development of DLY pigs.

68 Overall, we identified a large number of candidate SNP markers and ASE genes that may have a

69 contribution on DLY three-way crossbreeding system. These may be valuable for pig breeding

70 and production in the future.

71 Materials and Methods

72 All experimental procedures and sample collection methods in this study were approved by the

73 Institutional Animal Care and Use Committee (College of Animal Science and Technology of

74 Sichuan Agricultural University, Sichuan, China; approval number: No. DKY-B20121406).

75 Genome Sequencing Data of Pigs

76 In this study, we first downloaded genome sequencing data of 30 pig individuals (including 11

77 Duroc, 9 Landrace and 10 Yorkshire pigs) with mean genome coverage of $\sim 15.47 \times$ for each

78 individual (Supplementary Table S1). We then sequenced a DLY pig family, including two

79 grandparents (a male Landrace pig and a female Yorkshire pig), two parents (a male Duroc pig

80 and a female LY crossbred pig) and six offspring (three male and three female DLY crossbred 
81 pigs) using Illumina HiSeq 4000 platform (Supplementary Table S2). In total, we generated

$82 \sim 962.46 \mathrm{~Gb}$ paired-end 100-bp (PE100) high quality sequencing data for 10 pig individuals, with

83 mean genome coverage of $\sim 38.51 \times$ for each individual (Supplementary Table S2).

\section{$84 \quad$ SNP Calling}

85 The genome sequencing data of pigs in this study were first mapped to reference pig genome

86 (v.11.1, see URLs) using BWA (v.0.7.8) with default options (Li \& Durbin 2009). We used

87 "MarkDuplicates" module in package Picard (v.1.48, see URLs) to remove duplicated reads. The module "HaplotypeCaller" in Genome Analysis Toolkit (GATK; v.3.7) (McKenna et al., 2010) was used to call SNPs, which were next filtered by following criteria: QUAL $<30.0$, QD $<2.0$, $\mathrm{MQ}<40.0$, FS $>$ 60.0. Sex chromosomes (X and Y) were excluded for SNP calling. To further improve the accuracy of SNP calling, we empirically depleted $\sim 2 \%$ SNPs located in left and

92 right tails, based on the distribution of SNP depth (Supplementary Figure S2). Finally, we 93 identified a total of $\sim 12.56$ million (M) SNPs of three pig breeds (Supplementary Table S1) as

94 well as $\sim 13.29$ M SNPs of the DLY pig family (Supplementary Table S2). We used Illumina's porcine $60 \mathrm{~K}$ Genotyping Bead-Chip (v.2) to validate the accuracy of SNP calling for each 
97 (Purcell et al., 2007) was used to calculate the mendelian error to confirm the accurate kinship of

98 the DLY pig family.

99 Calculation of the Probability of Heterozygous SNP

100 To calculate the probability of heterozygous SNP (PHS) of simulated offspring generated by

101 different crossbreeding systems, we first calculated the frequency of three genotypes

102 (homozygous identical with reference, homozygous distinct from reference, heterozygous) for

103 each SNP locus based on the $\sim 12.56 \mathrm{M}$ population-scale SNPs for Duroc (11 individuals),

104 Landrace (9 individuals) and Yorkshire (10 individuals) respectively. We then simulated

105 crossbreeding between each two pig breeds and recalculated the frequency of the same three

106 genotypes for simulated offspring. Based on this method, we could obtain PHS for each SNP

107 locus of the simulated offspring generated by different crossbreeding systems.

108

109

110

111

112

\section{Principal Component Analysis and Function Enrichment Analyses}

We performed principal component analysis with software GCTA (v.1.91.3beta, see URLs)

based on the $\sim 12.56 \mathrm{M}$ population-scale SNPs. Function enrichment analyses were performed using the online toolkit "Metascape" with parameters "Min Overlap=3, $P$ Value Cutoff $=0.01$, Min Enrichment=1.5" (Zhou et al., 2019). 


\section{Identification of ASE Genes}

114 For each DLY individual, RNA was extracted from adipose, heart, liver and skeletal muscle.

115 RNA-Seq libraries were prepared from total RNA using poly(A) enrichment of the mRNA to

116 remove ribosomal RNA (rRNA). All RNA-Seq libraries were sequenced using an Illumina

117 HiSeq4000 platform, generating a total of $\sim 111.52 \mathrm{~Gb}$ transcriptome data (Supplementary

118 Figure S3). After quality control, high-quality transcriptome data were mapped to reference pig

119 genome (v11.1, see URLs) using STAR software (v.2.6.0) (Dobin et al., 2013) with parameters

“--outSAMattributes NH HI NM MD --alignEndsType EndToEnd” (Supplementary Figure

S4A). The heterozygous SNPs of each DLY individual were phased via trio genotypes and only

the phased exonic SNPs were used for further analyses. Reads covering multiple SNPs were

trimmed to leave one SNP locus so that a read only can be counted once, a necessary step for statistical analysis. SAMtools (Li et al., 2009) was then used to generate a pileup file, which were used to calculate the total number of reads aligning to each allele (Supplementary Figure

S4A). The total number of reads mapped to each gene for different alleles were summed up and 
129 allelic biases from the expected 1:1 distribution for allele-specific expression for each sample

130 (Supplementary Figure S4A). $P$-values were adjusted by bonferrioni method (Supplementary

131 Figure S5). Each gene was then classified to three categories, including ASE (adjust- $P<0.05$,

132 binomial test), NA (not significant for allele-specific expression), NHS (no heterozygous SNP)

133 (Supplementary Figure S4A). A high-confidence ASE gene should include two ASE categories

134 across the six DLY individuals (Supplementary Figure S4B).

135 URLs

136 Reference pig genome, ftp://ftp.ensembl.org/pub/release92/fasta/susscrofa/dna/Sus_s

137 crofa.Sscrofa11.1.dna.toplevel.fa.gz; Picard, http://picard.sourceforge.net; GCTA,

138 http://www.gcta-ga.org;

139 Results

140 Distribution of Heterozygous SNPs in Three-way Crossbred Pigs

141 To estimate the distribution of heterozygous SNPs in three-way crossbred pigs, we calculated the

142 PHS for simulated offspring generated by three kinds of three-way crossbreeding systems,

143 namely, Duroc $\times($ Landrace $\times$ Yorkshire $)($ DLY $)$, Landrace $\times($ Yorkshire $\times$ Duroc $)($ LYD $)$ and

144 Yorkshire $\times($ Duroc $\times$ Landrace $)($ YDL $)($ Figure 1A-F $)$. This was based on the $\sim 12.56$ M SNPs 
145 of the publicly available genome sequencing data of 30 pig individuals from three purebreds

146 (including 11 Duroc, 9 Landrace, and 10 Yorkshire pigs) with mean genome coverage of

$147 \sim 15.47 \times$ for each individual (Supplementary Table S1). Compared with the other two kinds of

148 three-way crossbreeding systems, DLY significantly exhibited more high-probability

149 heterozygous SNPs (Figure 1G, mean Pearson's $r=-0.99, P<0.01$ ). This was most likely

150 contributed to the closer genetic relationship between L and Y (Figure 1H). As a result,

151 compared with the other two kinds of two-way crossbreeding systems (Supplementary Figure

152 S1A), LY significantly exhibited fewer high-probability heterozygous SNPs (Supplementary

153 Figure S1B, mean Pearson's $r=-0.93, P<0.01$ ). Taking together, we suggest that DLY is a

154 more effective strategy for three-way crossbreeding system among Duroc, Landrace, and

155 Yorkshire breeds as it could yield more stably inherited heterozygous SNPs.

\section{Validation of the Accuracy of PHS}

157 To validate the accuracy of PHS, we sequenced a DLY pig family, including two grandparents (a

158 male Landrace and a female Yorkshire), two parents (a male Duroc and a female LY crossbred

159 individual) and six offspring (three male and three female DLY crossbred individuals) with mean

160 genome coverage of $38.51 \times$ for each individual (Supplementary Table S2). A total of $\sim 13.29 \mathrm{M}$ 
161 SNPs were identified, which covered $\sim 74.11 \%$ of the SNPs identified from the three pig

162 purebreds as well as $\sim 98.22 \%$ of the homozygous SNPs and $\sim 98.36 \%$ of the heterozygous SNPs

163 identified from Illumina's Porcine 60K Genotyping Bead-Chip (v.2) for each individual

164 (Supplementary Table S2 and Table S3). As expected, the six DLY pigs exhibited a larger

165 number of heterozygous SNPs (ranged from 6.12 M to 6.26 M) than the other four pigs (ranged

166 from 4.44 M to 5.62 M) (Supplementary TableS2). The percentage of heterozygous SNPs of

167 each pig individual was calculated for 20 equal intervals (based on PHS of simulated DLY

168 offspring, from $0 \%$ to $100 \%$, at intervals of 5\%) (Figure 1I). We found that the distribution of

169 simulated DLY offspring was significantly similar with the six DLY pigs (mean Pearson's $r=$

$1700.98, P<0.01$ ), but significantly different with the other four pigs (mean Pearson's $r=-0.39, P$

$171<0.01)$ (Figure 1I). This result strongly confirmed the accuracy of PHS and supported our

172 findings above.

173 Function Analyses of High-probability Heterozygous SNPs in DLY pigs

174 Annotation of the 14,848 high-probability heterozygous SNPs in DLY pigs (PHS > 0.9) using

175 ANNOVAR software (Wang et al., 2010) and Sus scrofa ENSEMBL gene annotation (v.92)

176 revealed that more than half $(\sim 59.68 \%)$ of the loci were enriched in intergenic regions (Figure 
177 2A), suggesting that a large fraction of the high-probability heterozygous SNPs were acted in

178 regulatory regions of the genome (Carneiro et al., 2014). The majority $(\sim 93.72 \%)$ of the left loci

179 were enriched in intronic regions, remaining $\sim 6.28 \%$ located in upstream, downstream, UTR and

exonic regions (Figure 2A). We found none of the coding SNPs was a nonsense, suggesting that

gene loss may not play a major role for breed-of-origin effect on DLY pigs. Only six genes

(AHI1, AKAP9, C8H4orf54, ENDOU, USP20, ZNF507) were identified to harbor a

nonsynonymous SNP locus (Figure2B), indicating that very few loci had the power to

completely alter protein-coding genes.

We further tested whether the six genes may have a close association with the crossbred

performance. AHII is required for both cerebellar and cortical development and has been

previously shown to be associated with fat development and obesity via regulating insulin signaling (Niu et al., 2012). $A K A P 9$ is a member of structurally diverse proteins that have the common function of binding to the regulatory subunit of protein kinase A (PKA) (Jo et al., 2016; 
193

194

195

196

197

198

199

200

201

202

203

204

205

206

207

208

Weyden et al., 2011).

\section{Identification of ASE Genes}

To investigate the breed-of-origin effects from purebreds on gene expression pattern during the development of DLY pigs, we sequenced four representative tissues, including adipose, heart, liver and skeletal muscle (Supplementary Figure S3) and sought to identify ASE genes for each tissue. Firstly, a large fraction (ranged from $\sim 88.59 \%$ to $\sim 89.59 \%$ ) of heterozygous SNPs for each DLY pig individual were phased via trio genotypes, that is, when at least one member of the trio was homozygous for the reference or alternate allele (Iliadis et al., 2012; Lajugie et al.,

2013) (Figure 3A-G). ASE genes were then identified based on these phased SNPs across the six DLY pigs for each tissue (Supplementary Figure 4A, B). In total, we identified 95, 79, 132 and 42 ASE genes in adipose, heart, liver and skeletal muscle, respectively, with a median value of allelic ratio ranged from 0.68 to 0.82 (Figure 4A, Supplementary File S2). Notably, we observed that $\sim 78.09 \%$ (196/251) of ASE genes were only detected in one tissue (Figure 4B), indicating that ASE genes were mainly tissue-specific, consistent with the results of principal component analysis (Figure 4C) and unrestricted cluster analysis (Figure 4D) based on gene expression of the 251 nonredundant ASE genes. 


\section{Discussion}

210

In this study, we investigated the breed-of-origin effects from purebreds on DLY three-way

211

crossbred pigs in genomic and transcriptional levels. In total, we identified 251 ASE genes

212

across adipose, heart, liver and skeletal muscle. To further explore the characteristics of these

213

ASE genes, we made a comparison with ASE genes reported in previous studies for pigs

214

(Esteve-Codina et al., 2011; Maroilley et al., 2017; Oczkowicz et al., 2018). We found none of

the ASE genes was shared across the four datasets, suggesting that ASE gene may be tissue-

specific or breed-specific (Supplementary Figure S8). Among the 251 nonredundant ASE

genes, only $38(\sim 15.14 \%)$ were covered by other datasets, remaining $213(\sim 84.76 \%)$ were first

reported in this study (Supplementary Figure S8).

219

The metabolism of adipose is deeply associated with pork production and quality, which are

220

the two major commercial traits for pig industries. In adipose, the top 3 significant GO pathways

221

of ASE genes include 'fatty acid metabolic process' $\left(P=7.24 \times 10^{-9}\right)$, 'acylglycerol metabolic

222 process' $\left(P=6.31 \times 10^{-8}\right)$ and 'regulation of cholesterol metabolic process' $\left(P=8.13 \times 10^{-7}\right)$

223 (Supplementary Figure S9A, Table S4), suggesting that ASE genes may play important roles

224 in adipose development for DLY pigs. For example, $F A B P$ is supposed to participate in 
metabolism of long-chain fatty acids and has been proved to have a close relationship with ligase family and functions in converting free long-chain fatty acids into fatty acyl-CoA esters; a previous study suggested this gene might contribute to the capacity of fat deposition and meat quality in pig breeds (Li et al., 2012). metabolites, like proteins, fatty acids, carbohydrates, drugs and bile acids. In liver, ASE genes were enriched in $18 \mathrm{GO}$ and KEGG pathways, such as 'Glutathione metabolism' $\left(P=2.88 \times 10^{-8}\right)$, 'steroid metabolic process' $\left(P=1.82 \times 10^{-7}\right)$, 'monocarboxylic acid metabolic process' $(P=$ $\left.2.95 \times 10^{-7}\right)$, 'small molecule catabolic process' $\left(P=2.82 \times 10^{-6}\right)$, 'Valine, leucine and isoleucine degradation' $\left(P=2.14 \times 10^{-5}\right)($ Supplementary Figure S9C, Table S6). 
241 calcium ion' $\left(P=4.07 \times 10^{-3}\right)$ and 'Tight junction' $\left(P=5.89 \times 10^{-3}\right)($ Supplementary Figure

242 S9B,D, Table S5, S7).

243 Taken together, we speculate that ASE genes may play important roles for the development

244 of DLY pigs, indicating the potential influence of breed-of-origin effects from purebreds on gene

245 expression of crossbred individuals.

\section{Conclusion}

247 In this study, we simulated three kinds of three-way crossbreeding system among Duroc,

248 Landrace and Yorkshire breeds, and estimated the distribution of heterozygous SNPs in terminal

249 crossbred pigs via whole genome sequencing data from three purebreds. We found DLY three-

250 way crossbreeding system could yield more stably inherited heterozygous SNPs in the terminal

251 crossbred offspring, suggesting that DLY is a more effective strategy for three-way

252 crossbreeding. We also sequenced a DLY pig family and identified a lot of ASE genes in adipose,

253 heart, liver and skeletal muscle. These ASE genes exhibited a tissue-specific pattern and were

254 involved in many important functional pathways, indicating the potential influence of breed-of-

255 origin effects from purebreds on gene expression of DLY crossbred pigs. These discoveries may

256 provide a valuable resource for pig breeding and production in the future and set an excellent 
257 model of crossbreeding analysis for other agricultural animals.

258

259 Abbreviation

260 ASE: allele-specific expression; SNP: single-nucleotide polymorphisms; PHS: probability of

261 heterozygous SNP; DLY: Duroc $\times($ Landrace $\times$ Yorkshire $) ;$ LYD: Landrace $\times($ Yorkshire $\times$

262 Duroc $)$; YDL: Yorkshire $\times($ Duroc $\times$ Landrace $)$; PCA: principal component analysis; QUAL:

263 variant quality; QD: variant confidence / quality by depth; MQ: mapping quality; FS: Phred-

264 scaled p-value using Fisher's exact test to detect strand bias.

265 Data Availability

266 The whole-genome sequencing data of ten pig individuals and transcriptome data of the six DLY

267 pig individuals of the DLY family are accessible at NCBI BioProject under accession number of

268 PRJNA507853. The genotyping data of the Illumina's porcine 60K Genotyping Bead-Chip (v.2)

269 have been submitted to the NCBI Gene Expression Omnibus (GEO) under accession number of

270 GSE123327.

\section{Author Contributions}

272 Yu.L and MZL conceived and designed the study. JDM, LJ, XW, MNH, KRL, ZQH, XWL and

273 YRG performed the experiments. Yu.L, QZT and Yan.L analyzed all the experiments. Yu.L and 
274 MZL drafted the manuscript. MZL, QZT revised the manuscript. All authors read and approved

275 the final manuscript.

276 FUNDING

277 This work was supported by grants from the National Key R \& D Program of China 278 (2018YFD0500403), the National Natural Science Foundation of China(31802044, 31872335

279 and 31772576), the Sichuan Province \& Chinese Academy of Science of Science \& Technology

280 Cooperation Project (2017JZ0025), the Science \& Technology Support Program of Sichuan

281 (2016NYZ0042 and 2017NZDZX0002), the Earmarked Fund for China Agriculture Research

282 System (CARS-35-01A) and the China Postdoctoral Science Foundation (2018M643514).

283 Competing Interests

284 The authors declare that they have no competing interests.

285 Reference

286 Andergassen D, Dotter CP, Kulinski TM, Guenzl PM, Bammer PC, Barlow DP, Pauler FM, Hudson QJ. 2015. Allelome.PRO, a pipeline to define allele-specific genomic features from high-throughput sequencing data. Nucleic Acids Research 43:e146 DOI 10.1093/nar/gkv727 
290

291

292

293

294

295

296

297

298

299

300

301

302

303

304

305

Andergassen D, Dotter CP, Wenzel D, Sigl V, Bammer PC, Muckenhuber M, Mayer D, Kulinski

TM, Theussl HC, Penninger JM, Bock C, Barlow DP, Pauler FM, Hudson QJ. 2017.

Mapping the mouse Allelome reveals tissue-specific regulation of allelic expression. Elife

6 DOI 10.7554/eLife. 25125

Carneiro M, Rubin CJ, Di Palma F, Albert FW, Alfoldi J, Martinez Barrio A, Pielberg G, Rafati

N, Sayyab S, Turner-Maier J, Younis S, Afonso S, Aken B, Alves JM, Barrell D, Bolet G,

Boucher S, Burbano HA, Campos R, Chang JL, Duranthon V, Fontanesi L, Garreau H,

Heiman D, Johnson J, Mage RG, Peng Z, Queney G, Rogel-Gaillard C, Ruffier M, Searle

S, Villafuerte R, Xiong A, Young S, Forsberg-Nilsson K, Good JM, Lander ES, Ferrand

N, Lindblad-Toh K, Andersson L. 2014. Rabbit genome analysis reveals a polygenic

basis for phenotypic change during domestication. Science 345:1074-1079 DOI

$10.1126 /$ science. 1253714

Chen K, Baxter T, Muir WM, Groenen MA, Schook LB. 2007. Genetic resources, genome mapping and evolutionary genomics of the pig (Sus scrofa). International Journal of Biological Sciences 3:153-165.

Cho KH, Kim MJ, Jeon GJ, Chung HY. 2011. Association of genetic variants for FABP3 gene 
306

307

308

309

310

311

312

with back fat thickness and intramuscular fat content in pig. Molecular Biology Reports 38(3):2161-6 DOI 10.1007/s11033-010-0344-3.

Choi YS, Lee JK, Jung JT, Jung YC, Jung JH, Jung MO, Choi YI, Jin SK, Choi JS. 2016. Comparison of Meat Quality and Fatty Acid Composition of Longissimus Muscles from Purebred Pigs and Three-way Crossbred LYD Pigs. Korean Journal for Food Science of Animal Resources 36:689-696 DOI 10.5851/kosfa.2016.36.5.689

Dobin A, Davis CA, Schlesinger F, Drenkow J, Zaleski C, Jha S, Batut P, Chaisson M, Gingeras TR. 2013. STAR: ultrafast universal RNA-seq aligner. Bioinformatics 29:15-21 DOI 10.1093/bioinformatics/bts635.

Esteve-Codina A, Kofler R, Palmieri N, Bussotti G, Notredame C, Pérez-Enciso M. 2011. Exploring the gonad transcriptome of two extreme male pigs with RNA-seq. $B M C$ Genomics 12:552 DOI 10.1186/1471-2164-12-552.

Gardin A, White J. 2011. The Sanger Mouse Genetics Programme: High Throughput Characterisation of Knockout Mice.

Hua LZ, Wu YZ, Bai FF, William KK, Feng ZX, Liu MJ, Yao JT, Zhang X, Shao GQ. 2014. Comparative analysis of mucosal immunity to Mycoplasma hyopneumoniae in 
322

323

324

325

326

327

328

329

330

331

332

333

334

335

336

337

Jiangquhai porcine lean strain and DLY piglets. Genetics and Molecular Research

13:5199-5206 DOI 10.4238/2014.July.7.13

Iliadis A, Anastassiou D, Wang X. 2012. A unified framework for haplotype inference in nuclear families. Annals of Human Genetics 76:312-325 DOI 10.1111/j.1469-1809.2012.00715.x

Jo YS, Kim MS, Yoo NJ, Lee SH. 2016. Frameshift Mutations of AKAP9 Gene in Gastric and Colorectal Cancers with High Microsatellite Instability. Pathology \& Oncology Research 22:587-592 DOI 10.1007/s12253-016-0042-0

Lajugie J, Mukhopadhyay R, Schizas M, Lailler N, Fourel N, Bouhassira EE. 2013. Complete genome phasing of family quartet by combination of genetic, physical and populationbased phasing analysis. PLoS ONE 8:e64571 DOI 10.1371/journal.pone.0064571

Li H, Durbin R. 2009. Fast and accurate short read alignment with Burrows-Wheeler transform. Bioinformatics 25:1754-1760 DOI 10.1093/bioinformatics/btp324

Li H, Handsaker B, Wysoker A, Fennell T, Ruan J, Homer N, Marth G, Abecasis G, Durbin R. 2009. The Sequence Alignment/Map format and SAMtools. Bioinformatics 25:20782079 DOI 10.1093/bioinformatics/btp352

Li Q, Tao Z, Shi L, Ban D, Zhang B, Yang Y, Zhang H, Wu C. 2012. Expression and genome 
polymorphism of ACSL1 gene in different pig breeds. Molecular Biology Reports 39(9):8787-92 DOI 10.1007/s11033-012-1741-6.

Liu X, Xiong X, Yang J, Zhou L, Yang B, Ai H, Ma H, Xie X, Huang Y, Fang S, Xiao S, Ren J, Ma J, Huang L. 2015. Genome-wide association analyses for meat quality traits in Chinese Erhualian pigs and a Western Duroc x (Landrace x Yorkshire) commercial population. Genetics Selection Evolution 47:44 DOI 10.1186/s12711-015-0120-x

Maroilley T, Lemonnier G, Lecardonnel J, Esquerré D, Ramayo-Caldas Y, Mercat MJ, RogelGaillard C, Estellé J. 2017. Deciphering the genetic regulation of peripheral blood transcriptome in pigs through expression genome-wide association study and allelespecific expression analysis. BMC Genomics 18(1):967 DOI 10.1186/s12864-017-4354-6

McKenna A, Hanna M, Banks E, Sivachenko A, Cibulskis K, Kernytsky A, Garimella K, Altshuler D, Gabriel S, Daly M, DePristo MA. 2010. The Genome Analysis Toolkit: a MapReduce framework for analyzing next-generation DNA sequencing data. Genome Research 20:1297-1303 DOI 10.1101/gr.107524.110

Niu S, Wang H, Huang Z, Rao X, Cai X, Liang T, Xu J, Xu X, Sheng G. 2012. Expression changes of hypothalamic Ahil in mice brain: implication in sensing insulin signaling. 
355

356

357

358

359

360

361

362

363

364

365

366

367

368

369

Oczkowicz M, Szmatoła T, Piórkowska K, Ropka-Molik K. 2018. Variant calling from RNA-seq data of the brain transcriptome of pigs and its application for allele-specific expression and imprinting analysis. Gene 641:367-375 DOI 10.1016/j.gene.2017.10.076.

Puppel K, Bogusz E, Golebiewski M, Nalecz-Tarwacka T, Kuczynska B, Slosarz J, Budzinski A, Solarczyk P, Kunowska-Slosarz M, Przysucha T. 2018. Effect of Dairy Cow Crossbreeding on Selected Performance Traits and Quality of Milk in First Generation Crossbreds. Journal of Food Science 83:229-236 DOI 10.1111/1750-3841.13988

Purcell S, Neale B, Todd-Brown K, Thomas L, Ferreira MA, Bender D, Maller J, Sklar P, de Bakker PI, Daly MJ, Sham PC. 2007. PLINK: a tool set for whole-genome association and population-based linkage analyses. The American Journal of Human Genetics 81:559-575 DOI 10.1086/519795

Ragab M, Sanchez JP, Minguez C, Baselga M. 2016. Crossbreeding effects on rabbit reproduction from four maternal lines of rabbits. Animal 10:1086-1092 DOI $10.1017 / \mathrm{s} 1751731115002918$

Rinell E, Heringstad B. 2018. The effects of crossbreeding with Norwegian Red dairy cattle on 
common postpartum diseases, fertility and body condition score. Animal 12:2619-2626

DOI $10.1017 / \mathrm{s} 175173111800037 \mathrm{x}$

372

373

374

375

376

377

Ruusunen M, Puolanne E, Sevon-Aimonen ML, Partanen K, Voutila L, Niemi J. 2012. Carcass and meat quality traits of four different pig crosses. Meat Science 90:543-547 DOI 10.1016/j.meatsci.2011.09.010

Sevillano CA, Vandenplas J, Bastiaansen JW, Calus MP. 2016. Empirical determination of breed-of-origin of alleles in three-breed cross pigs. Genetics Selection Evolution 48:55 DOI 10.1186/s12711-016-0234-9

Sheng Z, Pettersson ME, Hu X, Luo C, Qu H, Shu D, Shen X, Carlborg O, Li N. 2013. Genetic dissection of growth traits in a Chinese indigenous x commercial broiler chicken cross. BMC Genomics 14:151 DOI 10.1186/1471-2164-14-151

Stachowiak M, Szczerbal I, Flisikowski K. 2018. Investigation of allele-specific expression of genes involved in adipogenesis and lipid metabolism suggests complex regulatory mechanisms of PPARGC1A expression in porcine fat tissues. BMC Genetics 19(1):107 DOI 10.1186/s12863-018-0696-6

van der Weyden L, White JK, Adams DJ, Logan DW. 2011. The mouse genetics toolkit: 
revealing function and mechanism. Genome Biology 12:224 DOI 10.1186/gb-2011-12-6-

388

389

390

391

392

393

394

395

396

397

398

399

400

401

Vandenplas J, Calus MP, Sevillano CA, Windig JJ, Bastiaansen JW. 2016. Assigning breed origin to alleles in crossbred animals. Genetics Selection Evolution 48:61 DOI $10.1186 / \mathrm{s} 12711-016-0240-\mathrm{y}$

Venkatesh D, Mruk D, Herter JM, Cullere X, Chojnacka K, Cheng CY, Mayadas TN. 2016. AKAP9, a Regulator of Microtubule Dynamics, Contributes to Blood-Testis Barrier Function. The American Journal of Pathology 186:270-284 DOI 10.1016/j.ajpath.2015.10.007

Wang K, Li M, Hakonarson H. 2010. ANNOVAR: functional annotation of genetic variants from high-throughput sequencing data. Nucleic Acids Research 38:e164 DOI 10.1093/nar/gkq603

Wilhelmsson U, Stillemark-Billton P, Borén J, Pekny M. 2019. Vimentin is required for normal accumulation of body fat. Biological Chemistry 400(9):1157-1162 DOI 10.1515/hsz2019-0170 
402

403

404

405

406

407

408

409

410

411

412

413
Ponsuksili S, Li K, Mei, Liu G. 2015. Genome-wide identification of allele-specific expression in response to Streptococcus suis 2 infection in two differentially susceptible pig breeds. Journal of Applied Genetics 56(4):481-491 DOI 10.1007/s13353-015-0275-8.

Yang Y, Tang Z, Fan X, Xu K, Mu Y, Zhou R, Li K. 2018. ranscriptome analysis revealed chimeric RNAs, single nucleotide polymorphisms and allele-specific expression in porcine prenatal skeletal muscle. Scientific Reports 6:29039 DOI 10.1038/srep29039.

Zhang J, Chai J, Luo Z, He H, Chen L, Liu X, Zhou Q. 2018. Meat and nutritional quality comparison of purebred and crossbred pigs. Animal Science Journal 89:202-210 DOI 10.1111/asj.12878.

Zhou Y, Zhou B, Pache L, Chang M, Khodabakhshi AH, Tanaseichuk O, Benner C, Chanda SK. 2019. Metascape provides a biologist-oriented resource for the analysis of systems-level datasets. Nature Communication 10(1):1523 DOI 10.1038/s41467-019-09234-6 


\section{Figure 1}

Simulation of three-way crossbreeding system and validation of the accuracy of PHS.

A-F: Three kinds of simulated three-way crossbreeding systems among Duroc, Landrace, and Yorkshire pigs. G: The distribution of SNP number of simulated offspring of three kinds of three-way crossbreeding for 20 equal intervals (based on PHS, from $0 \%$ to $100 \%$, with intervals of 5\%). Pearson's $r$ was inferred between each two kinds of three-way crossbreeding systems. H: Principal component analysis (PCA) of the three pig breeds using $\sim 12.56 \mathrm{M}$ population-scale SNPs. I: Validation of PHS. Percentages of heterozygous SNPs of each pig for 20 equal intervals (based on PHS of simulated DLY offspring, from $0 \%$ to $100 \%$, with intervals of 5\%) were calculated. Pearson's $r$ was inferred for each comparison between simulated DLY offspring (red line) and sequenced pig individual. 
A

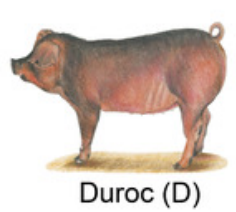

D

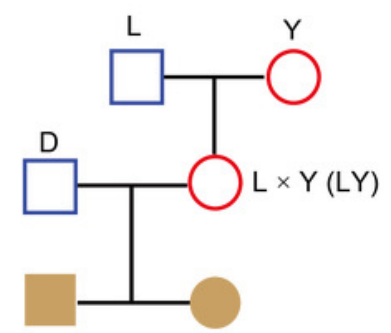

$D \times(L \times Y)(D L Y)$
B

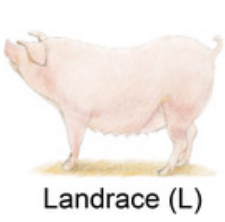

C

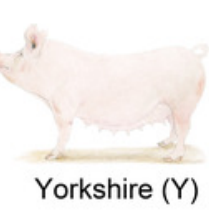

E

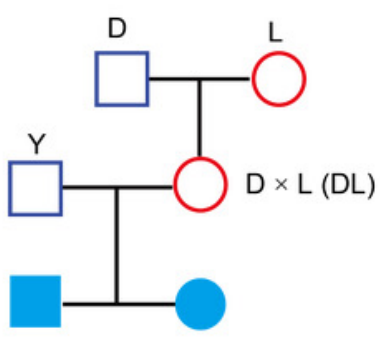

$\mathrm{Y} \times(\mathrm{D} \times \mathrm{L})(\mathrm{YDL})$
$\mathrm{F}$

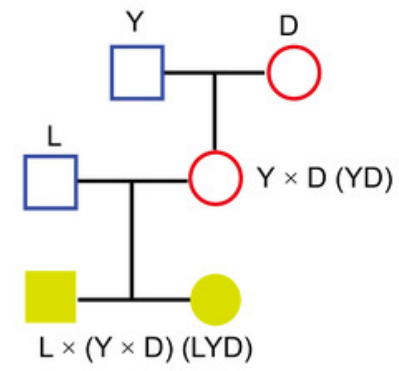

G

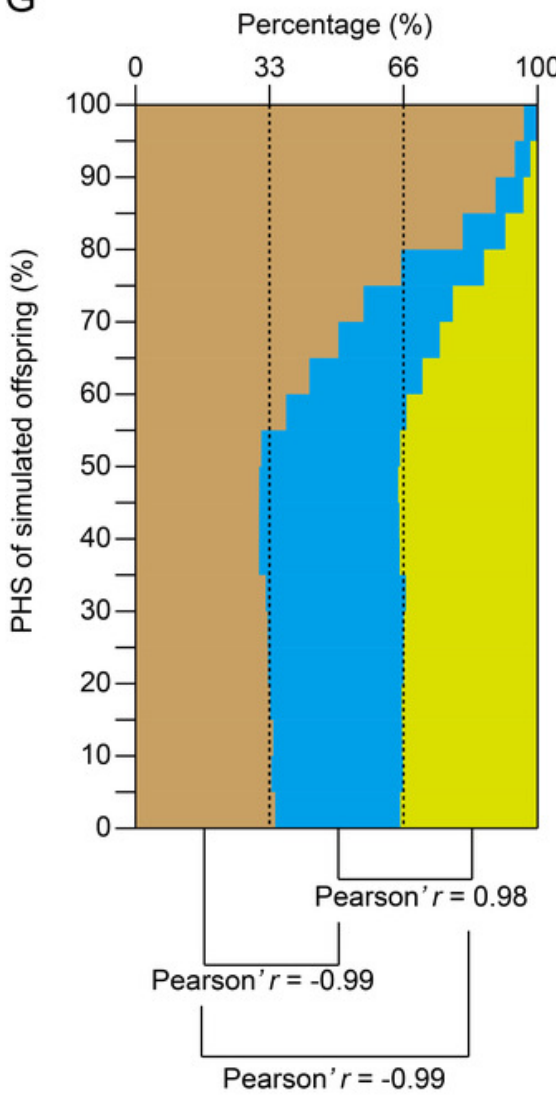

$\mathrm{H}$

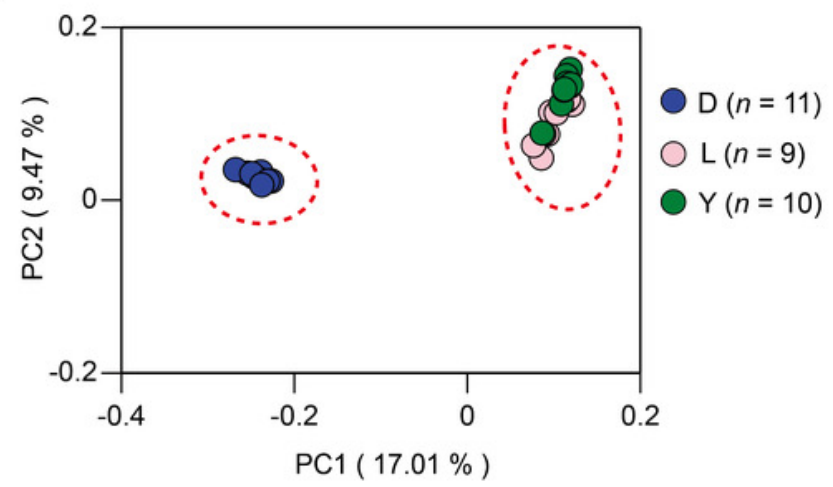

$4.74 \mathrm{M}$

$3.66 \mathrm{M}$

$3.42 \mathrm{M}$

$3.12 \mathrm{M}$

$3.13 \mathrm{M}$

$2.82 \mathrm{M}$

$2.74 \mathrm{M}$

$2.58 \mathrm{M}$

$1.92 \mathrm{M}$

$1.12 \mathrm{M}$

of SNPS

$.64 \mathrm{~K}$

$69.21 \mathrm{~K}$

$41.39 \mathrm{~K}$

$66 \mathrm{~K}$

$62.31 \mathrm{~K}$

$69 \mathrm{M}$

I

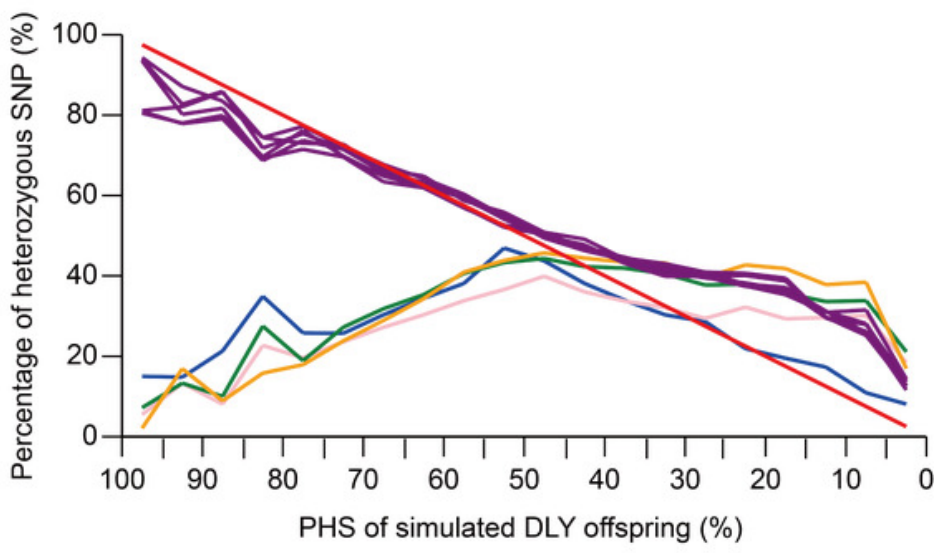

$\longrightarrow \mathrm{D}(n=1)-\mathrm{L}(n=1) \longrightarrow \mathrm{Y}(n=1)$
$\mathrm{LY}(n=1) \longrightarrow \mathrm{DLY}(n=6)$ 
Figure 2

Annotation of the 14,848 high-probability heterozygous SNPs in DLY pigs.

A: These SNPs were annotated using ANNOVAR software and classified to six genomic elements, including intergenic, intronic, UTR, downstream, upstream and exonic. B: The detailed information of the six genes that harbored a nonsynonymous SNP were listed.

A

Intergenic $(n=8,861$ ratio $=\sim 59.68 \%)$

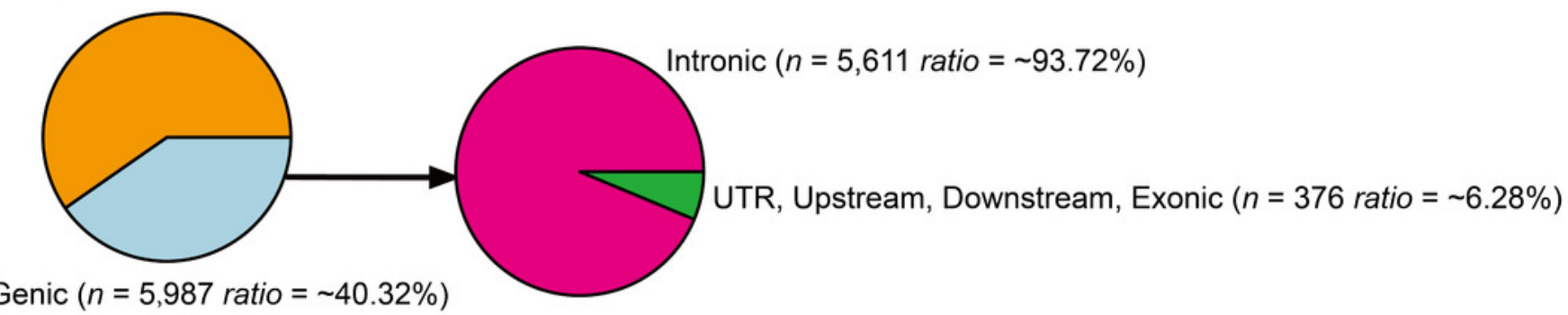

B

\begin{tabular}{llllll}
\hline Gene & Chromosome & Position & Genotype & $\begin{array}{l}\text { Amino acid } \\
\text { Substitution }\end{array}$ & $\begin{array}{c}\text { Number of } \\
\text { Heterozygous SNP }\end{array}$ \\
\hline AHI1 & 1 & $28,589,827$ & A / G & N196D & 6 \\
AKAP9 & 9 & $72,027,468$ & T/C & F3010S & 6 \\
C8H4orf54 & 8 & $120,797,165$ & T/G & I1496M & 6 \\
ENDOU & 5 & $78,083,854$ & A / G & M21T & 6 \\
USP20 & 1 & $270,017,455$ & T/C & L104S & 6 \\
ZNF507 & 6 & $42,281,876$ & G / A & D223N & 6 \\
\hline
\end{tabular}




\section{Figure 3}

SNP phasing of the six DLY pigs via trio genotypes.

A:The theory for phasing SNP using trio genotypes. For each heterozygous SNP locus of offspring, it could be inherited by parents based on seven conditions, among which two (refers to red, where parents are both homozygous but distinct) and four (refers to blue, homozygous for one parent and heterozygous for the other parent) can be used to phase.BG:The distribution of phased SNPs across autosomes of the six DLY pigs, ranged from $88.59 \%$ to $89.59 \%$. 
A

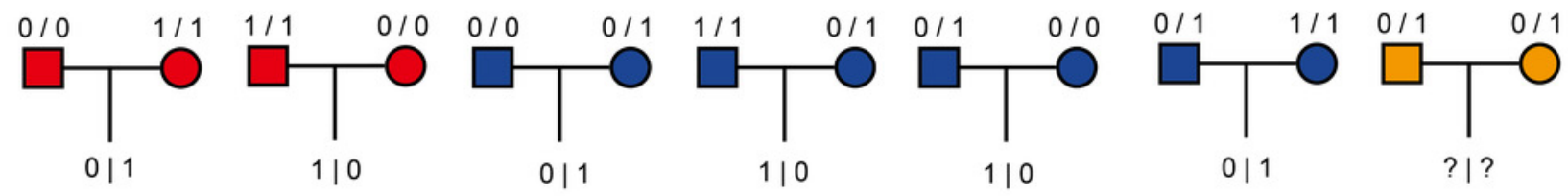

B

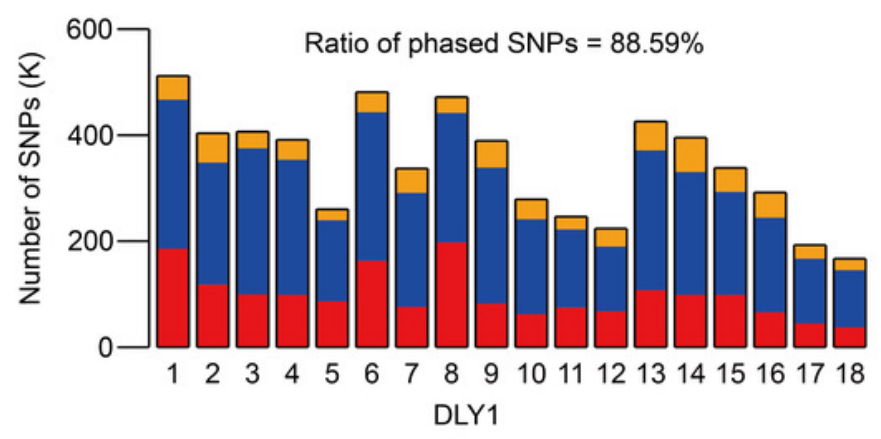

D

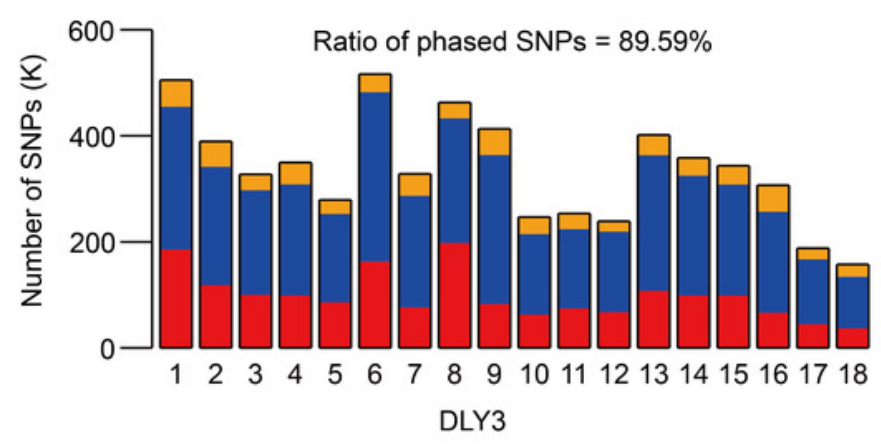

F

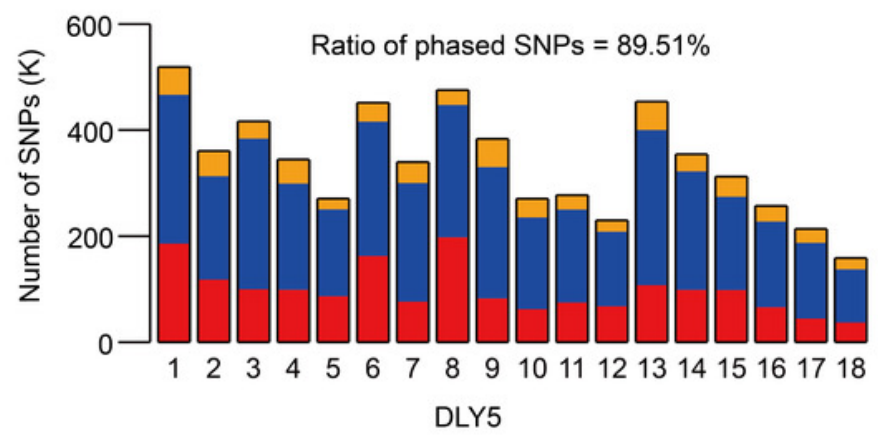

C

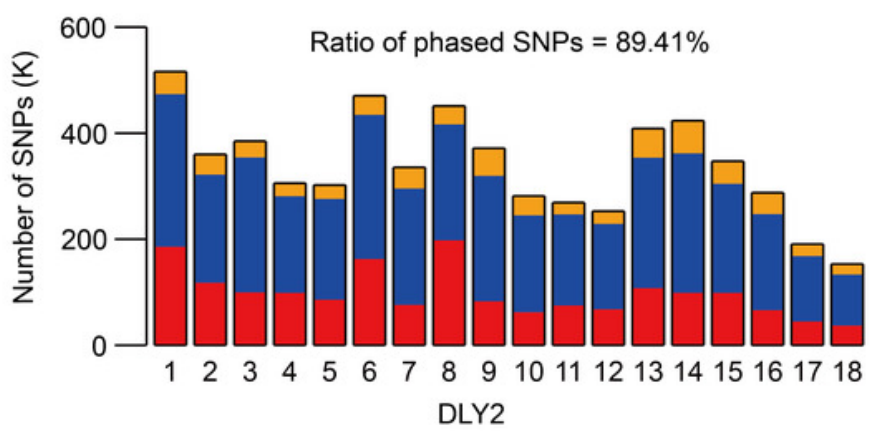

E

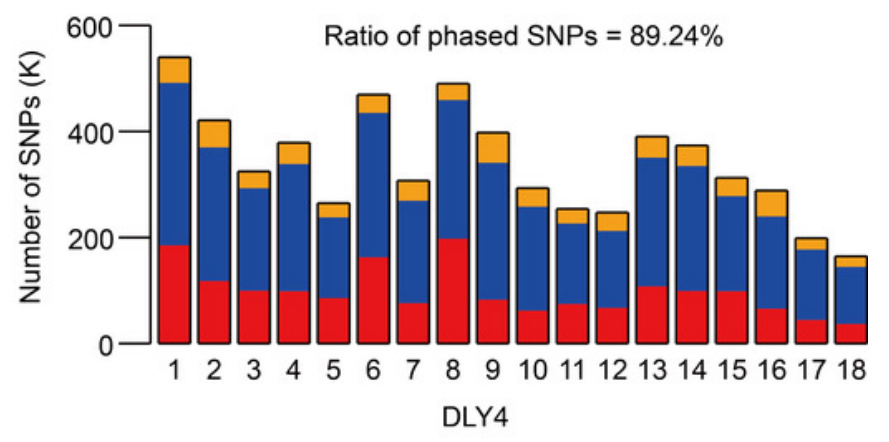

G

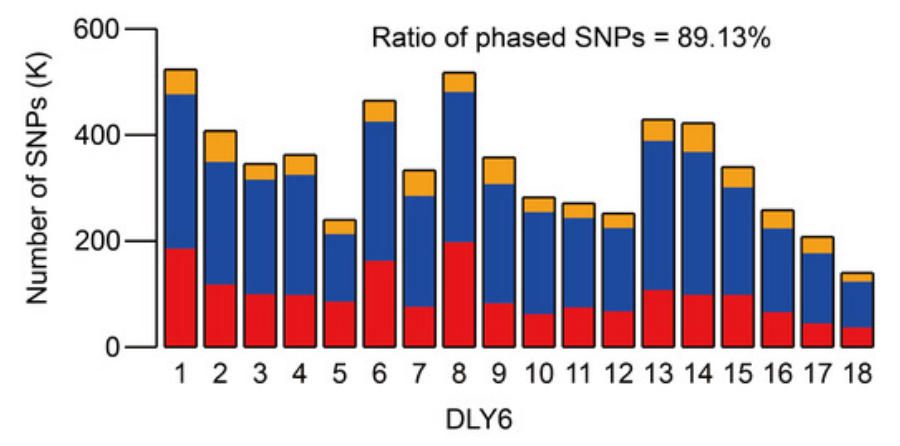


Figure 4

The results of ASE genes.

A:A total of 95, 79, 132 and 42 ASE genes were identified in adipose, heart, liver and skeletal muscle, with a median value of allelic ratio ranged from 0.68 to 0.82 . B:The venn diagram of ASE genes among adipose, heart, liver and skeletal muscle. C:Principal component analysis (PCA) of the 251 nonredundant ASE genes. D:Heatmap of the 251 nonredundant ASE genes.

A

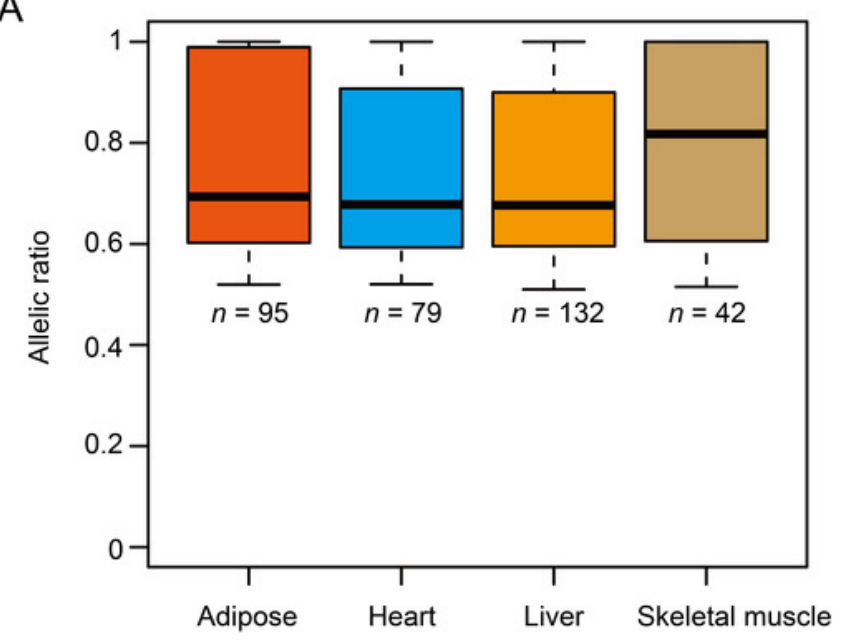

C

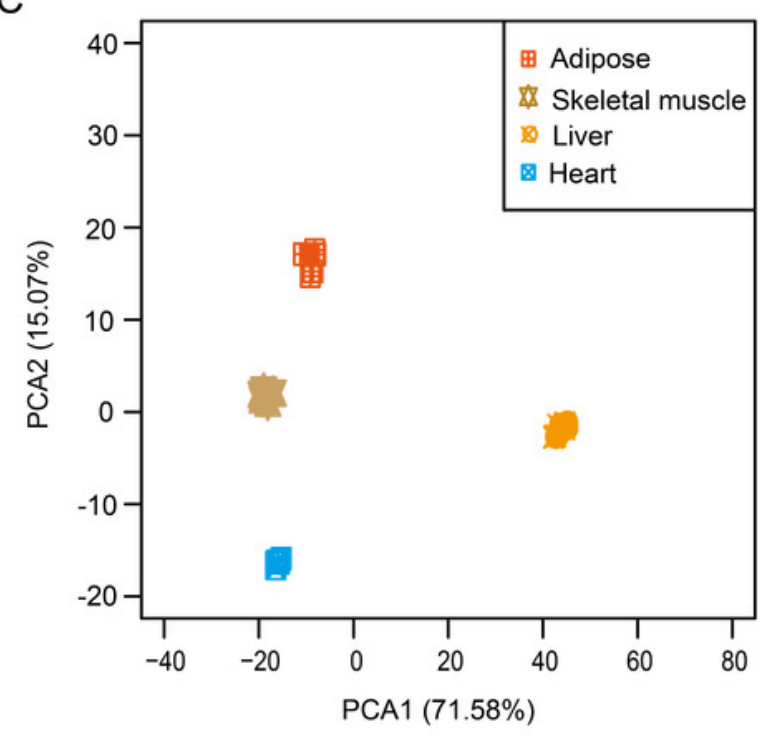

B
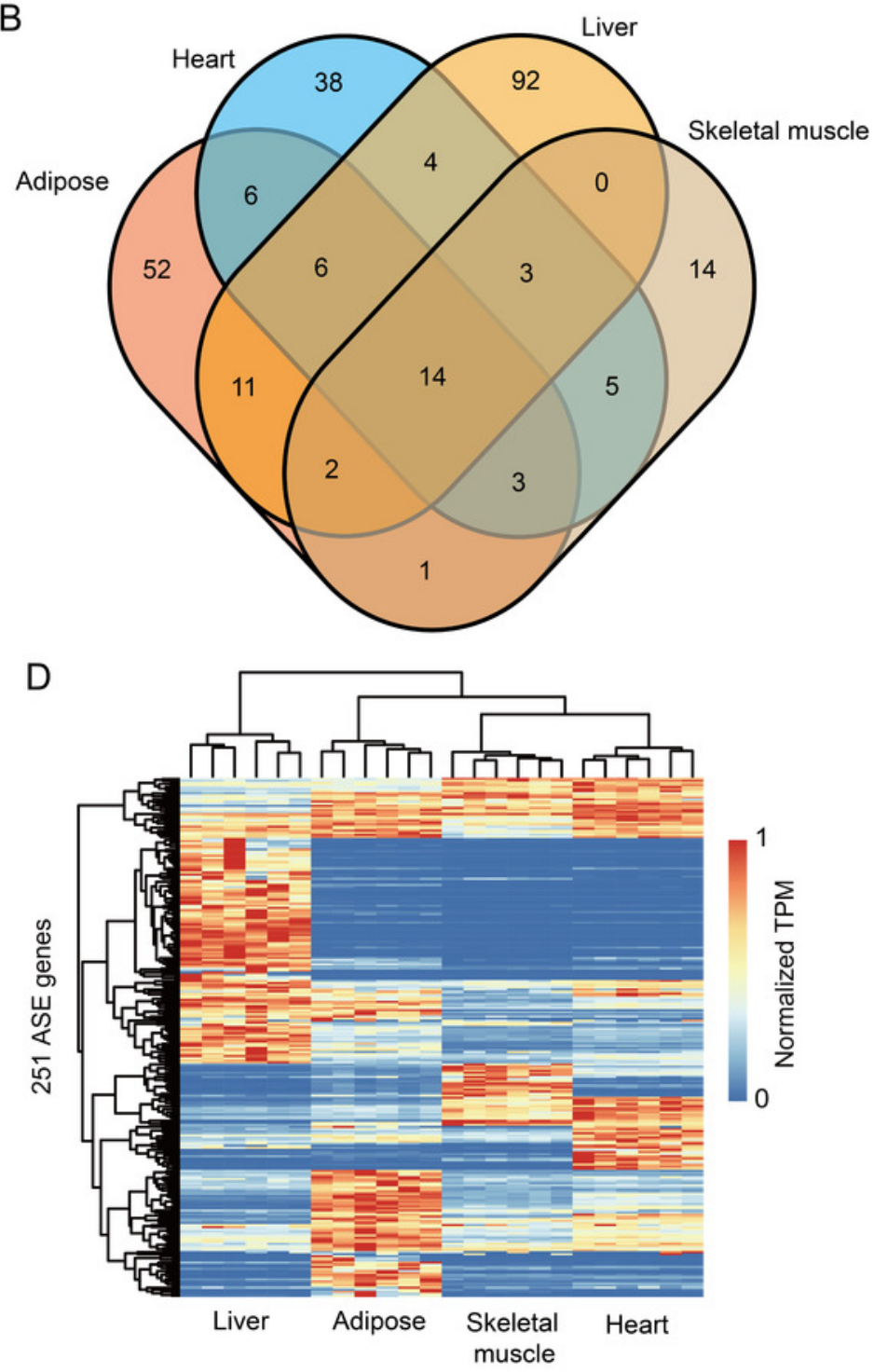